
\title{
25 Research Square \\ Transmission of gut bacteria and viruses from mothers to infants
}

\section{Sanzhima Garmaeva}

Department of Genetics,University of Groningen and University Medical Center Groningen https://orcid.org/0000-0002-0429-833X

\section{Trishla Sinha}

Department of Genetics, University of Groningen and University Medical Center Groningen, Groningen, The Netherlands https://orcid.org/0000-0002-0992-7983

\section{Anastasia Gulyaeva}

University Medical Center Groningen

\section{Ranko Gacesa}

University of Groningen and University Medical Center Groningen https://orcid.org/0000-0003-21190539

\section{Sergio Andreu-Sanchez}

University Medical Center Groningen https://orcid.org/0000-0002-3503-9971

\section{Arnau Vich Vila}

University of Groningen and University Medical Center Groningen https://orcid.org/0000-0003-46915583

\section{Lianmin Chen}

The First Affiliated Hospital of Nanjing Medical University

\section{Johanne Spreckels}

University Medical Center Groningen https://orcid.org/0000-0002-8711-1736

\section{Siobhan Brushett}

University of Groningen and University Medical Center Groningen

\section{Marloes Kruk}

Department of Genetics, University of Groningen and University Medical Center Groningen.

\section{Jackie Dekens}

Department of Genetics, University of Groningen and University Medical Center Groningen.

\section{Jan Sikkema}

University of Groningen and University Medical Center Groningen

\section{Folkert Kuipers}

University Medical Center Groningen https://orcid.org/0000-0003-2518-737X

\section{Andrey Shkoporov}

University College Cork https://orcid.org/0000-0002-5547-8672

\section{Colin Hill}


University College Cork https://orcid.org/0000-0002-8527-1445

\section{Sicco Scherjon}

University Medical Center Groningen

\section{Alexander Kurilshikov}

University of Groningen, University Medical Center Groningen https://orcid.org/0000-0003-2541-5627

\section{Cisca Wijmenga}

University Medical Centre Groningen https://orcid.org/0000-0002-5635-1614

\section{Jingyuan Fu}

University Medical Center Groningen https://orcid.org/0000-0001-5578-1236

Alexandra Zhernakova ( $\sim$ sashazhernakova@gmail.com)

Department of Genetics,University of Groningen and University Medical Center Groningen

\section{Article}

Keywords: infant gut virome, mother-to-infant transmission, metagenomes

Posted Date: November 29th, 2021

DOI: https://doi.org/10.21203/rs.3.rs-1079760/v1

License: (c) (1) This work is licensed under a Creative Commons Attribution 4.0 International License. Read Full License 


\section{Transmission of gut bacteria and viruses from}

\section{mothers to infants}

Sanzhima Garmaeva ${ }^{1 *}$, Trishla Sinha ${ }^{1 *}$, Anastasia Gulyaeva ${ }^{1}$, Ranko Gacesa ${ }^{1,2}$, Sergio Andreu-Sánchez ${ }^{1,3}$, Arnau Vich Vila ${ }^{1,2}$, Lianmin Chen $^{1}$, Johanne E Spreckels ${ }^{1}$, Siobhan Brushett $^{1,4}$, Marloes Kruk $^{1}$, Lifelines NEXT cohort study ${ }^{\S}$, Jackie Dekens ${ }^{1,5}$, Jan Sikkema ${ }^{5}$, Folkert Kuipers $^{3}$, Andrey Shkoporov ${ }^{6,7}$, Colin Hill $^{6,7}$, Sicco Scherjon ${ }^{8}$, Alexander Kurilshikov ${ }^{1}$, Cisca Wijmenga ${ }^{1}$, Jingyuan $\mathrm{Fu}^{1,3}$ and Alexandra Zhernakova ${ }^{1 \#}$

${ }^{1}$ Department of Genetics, University of Groningen and University Medical Center Groningen, Groningen, the Netherlands

${ }^{2}$ Department of Gastroenterology and Hepatology, University of Groningen and University Medical Center Groningen, Groningen, the Netherlands

${ }^{3}$ Department of Pediatrics, University of Groningen and University Medical Center Groningen, Groningen, the Netherlands

${ }^{4}$ Department of Health Sciences, University of Groningen and University Medical Center Groningen, Groningen, the Netherlands

${ }^{5}$ University Medical Center Groningen, Center for Development and Innovation

${ }^{6}$ APC Microbiome Ireland, University College Cork, Cork, Ireland

${ }^{7}$ School of Microbiology, University College Cork, Cork, Ireland

${ }^{8}$ Department of Obstetrics and Gynecology, University of Groningen and University Medical Center Groningen, Groningen, the Netherlands

* shared first authors: SG and TS

\# corresponding author: Alexandra Zhernakova 


\section{Abstract}

1 Seeding and development of the gut ecosystem are crucial for health, both in childhood and

2 later in life. While the composition of infant gut bacterial communities has been described,

3 the composition and origin of the infant gut virome remains under-studied. Here, we explore

4 mother-to-infant transmission of bacteria and viruses in 30 mother-infant pairs in a

5 longitudinal collection of faecal samples taken during pregnancy and the first 3 months after

6 birth. We demonstrate that infant bacterial strains resemble maternal strains more than

7 those of unrelated mothers. We quantify viromes using a complementary approach

8 examining both total metagenomes and viral metagenomes. The virome composition is highly

9 consistent between viral and total metagenomes. The infant gut viromes are dominated by

10 active temperate bacteriophages, which are more abundant in infants than mothers ( $p$ -

11 value=7.2e-06). We observe that the proportion of shared viruses between maternal and

12 infant gut is only $11.3 \%$ when considering the active virome fraction alone, but increases to

$13 \quad 37.6 \%$ when taking into account temperate phages in the form of prophages. These findings

14 indicate that viruses are vertically transmitted from mothers to infants early in life and that

15 pioneering phages can reach the infant gut via vertical transmission of their bacterial hosts.

\section{Introduction}

16 The human early life gut ecosystem has garnered much interest in recent years because of its

17 links to health and disease later in life ${ }^{1}$, but the core aspects of its origin and development

18 remain less understood. Studies have characterised the development of the infant gut

19 microbiome through the first 2-3 years of life, after which the gut microbiome reaches a state

20 of high microbial richness equivalent to that of an adult ${ }^{2,3}$. Thus far, the focus of research has been on the development of gut bacteria. However, the gut ecosystem also comprises viruses, 
archaea and eukaryotes such as fungi and protists, whose role in early gut ecosystem development has received less attention. Both vertical transmission (during pregnancy and birth) and horizontal transmission (from environmental sources like infant diet) have been described as sources of infant gut microbiota ${ }^{4}$. Recent studies provide increasing support for the maternal gut bacterial reservoir as a key source of microbial transmission from mothers to infants ${ }^{5,6}$ Although the transmission of infectious viruses such as cytomegalovirus and herpes simplex virus in the context of maternal and infant morbidity has been established ${ }^{7}$, little is known about the transmission of bacteriophages (bacterial viruses) from maternal to infant gut. This is partially due to difficulties in isolating and annotating metaviromes ${ }^{8}$. As environmental studies have clearly demonstrated that bacteriophages are key players in the modulation of bacterial communities $^{9,10}$, it is crucial to study them in the context of the developing human gut ecosystem as the bacterial community is established in the months following birth.

A recent study in 20 healthy infants using viral-like particle (VLP) data provided substantial functional evidence that the bacteriophages colonising the infant gut arise from excisions from pioneering infant gut bacteria ${ }^{11}$. However, the origin of these pioneering bacteriophages, as well as their hosts and their possible roots in the maternal gut ecosystem, remain underexplored. We therefore sequenced 183 total metagenomes (TMs) obtained by isolating all microbial DNA from stool and 66 viral metagenomes (VMs) using a VLP

41 enrichment isolation protocol from 30 mother-infant pairs for whom we have longitudinal samples collected during pregnancy and the first 3 months of life. We first described the bacterial and viral composition in both mothers and infants and elucidated the degree of inter-individual and intra-individual variability. We then compared bacterial strains and viruses across and within mother-infant pairs and found that infant bacterial strains resemble 
their mother's strains more than those of unrelated mothers. We found that the proportion

47 of shared active viruses within mother-infant pairs was lower than the proportion of shared

48 bacteria. However, we also found significantly more viruses that were shared between mother-infant pairs when taking into account bacteriophages incorporated in the bacterial genomes of maternal TMs, which indicates that maternal prophages are a source of pioneering infant gut bacteriophages.

\section{Results}

\section{Study sample and gut bacterial composition in mothers and infants}

We first characterised the gut bacteriome of mothers and infants and described the degree of sharedness of bacterial species in 183 TMs from 30 mothers and 32 infants, including 2 twin pairs (Figure 1a). Mothers collected their faeces at months 3 and 7 during pregnancy, at birth and at 3 months after birth (Figure 1a). Faecal samples from infants were collected at months 1, 2 and 3 after birth. Although meconium samples collected at birth were available, only 3 of the 32 meconium samples had a sufficient number of microbial reads, reflecting a low bacterial abundance in these samples, and these 3 meconium samples were therefore

60 excluded from the analysis. In this study, mothers had a median age of $32.0 \pm 4.4$ years, a median pre-pregnancy of $\mathrm{BMI}$ of $23.0 \pm 2.6 \mathrm{~kg} / \mathrm{m}^{2}$ (approximately 5 years prior to the pregnancy) and a median gestational age of $39.6 \pm 1$ week. The infants were $56 \%$ female and had a median birth weight of $3,705 \pm 380.4$ grams. $87.5 \%$ of the infants were vaginally delivered, with one twin pair having different delivery modes, and $28 \%$ were born at home. In terms of feeding, $53.6 \%$ were exclusively breastfed at month $1,56.3 \%$ were exclusively S1). 
The infant gut bacteriome showed a remarkably lower species-level alpha-diversity

69 compared to the maternal gut bacteriome (mean mother=2.62, mean infant=1.26, $p$ -

70 value=1.12e-34, (Supplementary Fig. 1a)), consistent with previous findings ${ }^{5}$. Over the entire

71 study period, maternal alpha-diversity did not show any significant association patterns to

72 maternal phenotypes or timepoint (Supplementary Fig. 1c), although this analysis may have

73 been hampered by low sample size. The alpha-diversity of bacterial species in infants also did

74 not change significantly during the first 3 months (Supplementary Fig. 1b). At the phylum

75 level, mothers and infants showed remarkably different compositions (Figure 1b, c), with

76 infants having more Actinobacteria and Proteobacteria and mothers having more

77 Bacteroidetes and Firmicutes (FDR<0.05) (Supplementary Table S2).

The species-level bacteriome composition was significantly different in mothers and

infants (permutational multivariate analysis of variance (PERMANOVA) $p$-value $=0.001,999$ permutations, Figure 1d). The maternal bacteriome showed greater inter-individual variation

81 than intra-individual variation across timepoints (Supplementary Fig. 2b). Infants also demonstrated more inter-individual variation than intra-individual variation (Supplementary

83 Fig. 2a). We next explored the relation between host phenotypes and the abundance of 84 microbial species and for example found that infants who were exclusively breastfed had a 85 significantly higher abundance of Cutibacterium acnes, a bacterium typically known to be a member of skin flora (FDR=0.001) (Supplementary Table S3). 

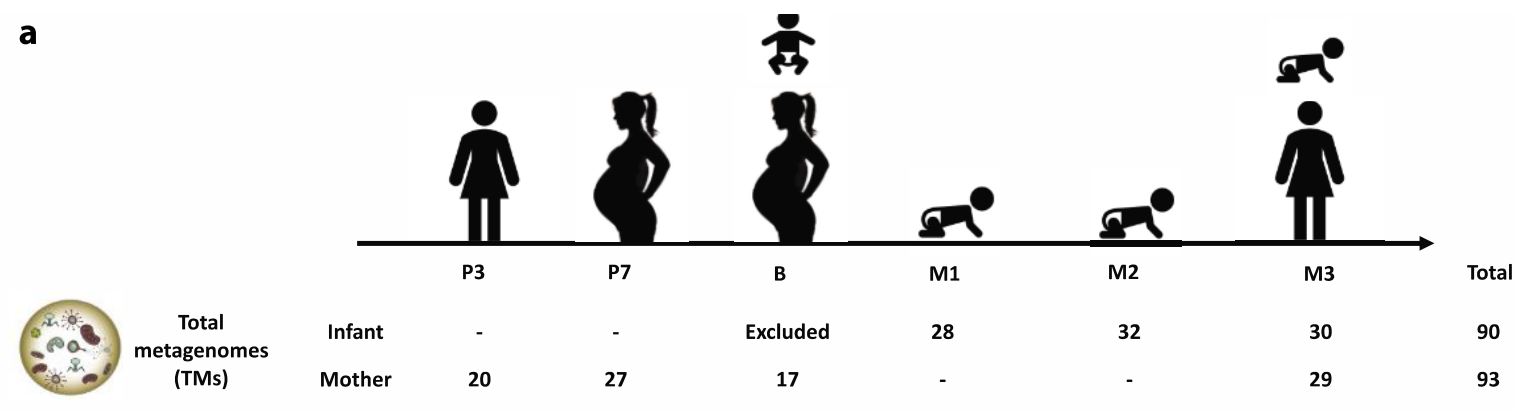

$\begin{array}{ccccccccc}\text { Viral } & \text { Infant } & - & - & - & 9 & 10 & 13 & 32 \\ \begin{array}{c}\text { metagenomes } \\ \text { (VMs) }\end{array} & \text { Mother } & - & 23 & 11 & - & - & - & 34\end{array}$
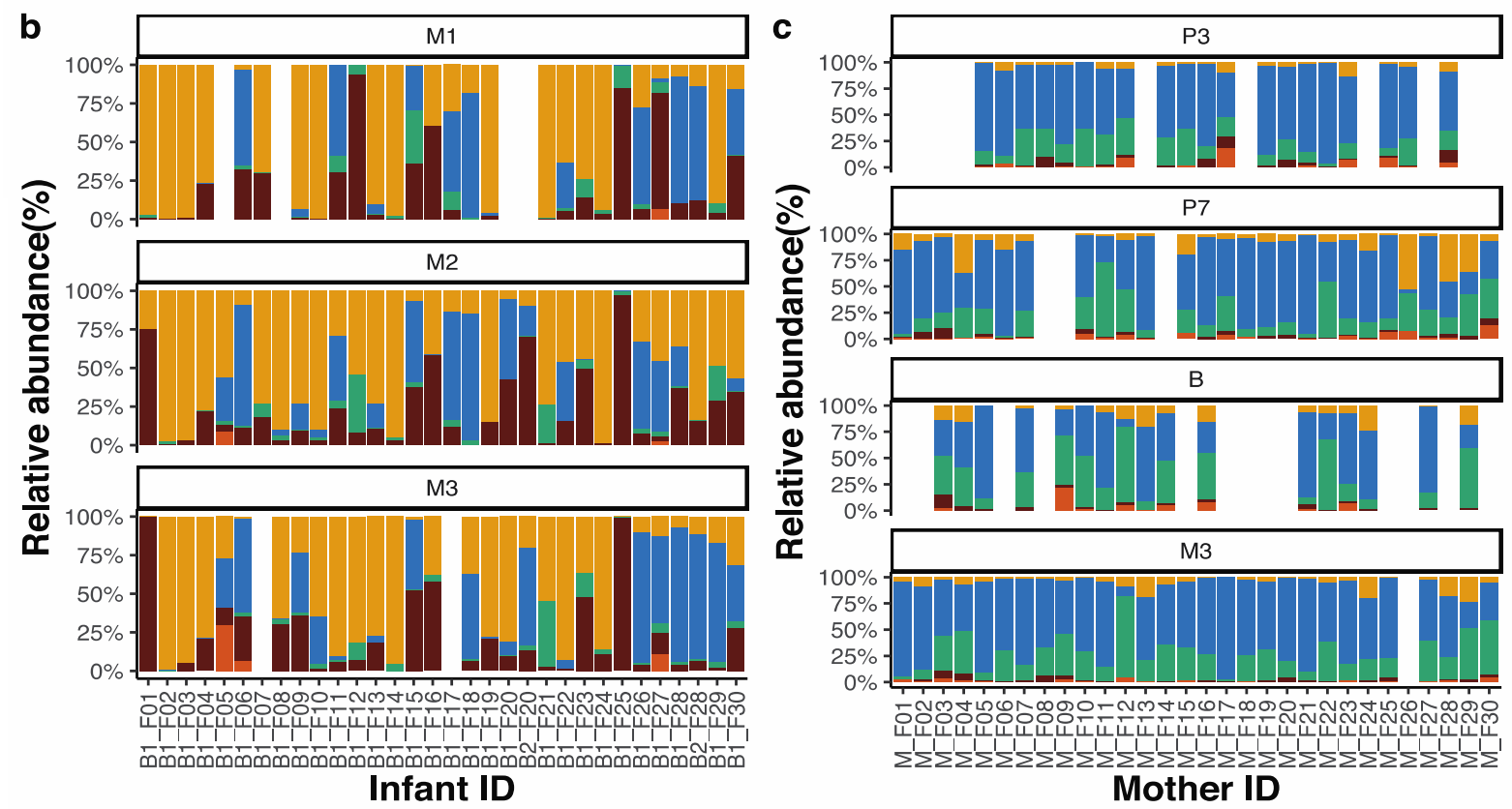

Phylum Actinobacteria $\quad$ Bacteroidetes

Firmicutes $\square$ Proteobacteria $\square$ Verrucomicrobia

d

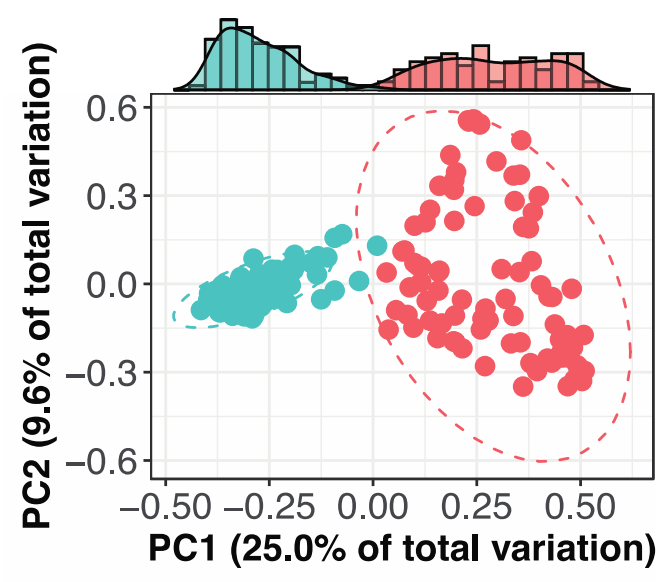

Type

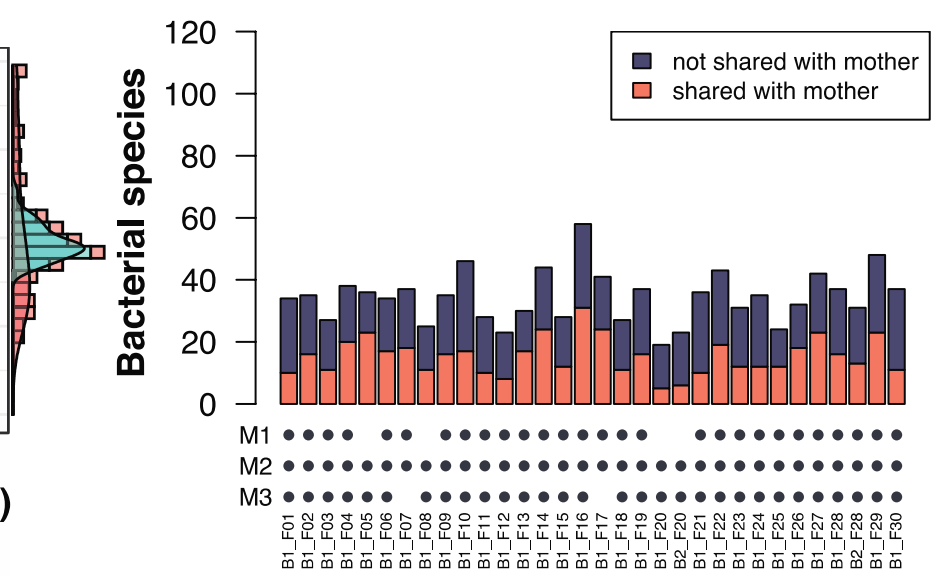

Infant

Figure 1: Study description and differences and sharedness in the bacterial composition between mothers and infants. 
a, Distribution of successfully sequenced samples across mothers and infants at month 3 (P3) and month 7 of pregnancy (P7), birth (B) and the first, second and third month after birth (M1, M2 and $M 3$, respectively). $\mathbf{b}-\mathbf{c}$, Phylum-level relative abundance profiles for infant (b) and maternal (c) samples. $\mathbf{d}, \mathrm{PCoA}$ analysis showing the differences in overall bacterial composition between mothers and infants calculated using Bray-Curtis dissimilarity matrix. Ellipses show 95\% confidence intervals. e, Number of the bacterial species in infants shared with own mother. Number of dots represents the number of available timepoints for each infant.

\section{Maternal to infant vertical transmission of bacteria}

87 Although microbiome composition differed between mothers and infants, $43.6 \pm 10.0 \%$ of bacterial species were shared across related mother - infant pairs (considering all timepoints in mothers and infants, Figure 1e, Supplementary Table S4). We next aimed to explore whether bacterial species shared between related mother-infant pairs are represented by the same strains. For 12 of the 17 species for which strain-level data could be constructed for both mothers and infants, we found at least one incidence of a mother-infant pair sharing an identical dominant strain. These species were Akkermansia muciniphila, Alistipes finegoldii, Bacteroides dorei, Bacteroides vulgatus, Bifidobacterium adolescentis, Bifidobacterium bifidum, Bifidobacterium longum, Bifidobacterium breve, Bilophila wadsworthia, Collinsella aerofaciens, Parabacteroides distasonis and Bifidobacterium uniformis. In 10 of these species (B. dorei, B. longum, A. finegoldii, B. uniformis, B. vulgatus, B. adolescentis, B. bifidum, B. wadsworthia, $C$. aerofaciens and $P$. distasonis), the phylogenetic distances between related mothers and infants were significantly lower than the distances between unrelated

100 individuals (999 permutations, FDR< 0.05, Figure 2c), suggesting either horizontal or vertical strain transmission of these species from mothers to infants. We hypothesised that birth mode and delivery location might affect dominant strain- 
104 showed a different pattern of transmission from mothers to infants depending on the place

105 of delivery (PERMANOVA, 999 permutations, $p=0.001$ ). For home deliveries (28\% of

106 deliveries), the B. bifidum phylogenetic distances between infants and their mothers were

107 small, whereas the B. bifidum phylogenetic distances between infants and mothers tended to

108 be larger for hospital deliveries (Figure 2a). Interestingly, in family 20, twin pairs shared

109 identical dominant strains of B. uniformis and B. breve despite having opposing modes of

110 delivery. Furthermore, we observed an interesting pattern in one family where the strain of

111 A. muciniphila showed a strain replacement during pregnancy, with infant strains being

112 similar to those of mother after birth in contrast to maternal strains just before birth (Figure

$1132 b)$.

a Bifidobacterium bifidum

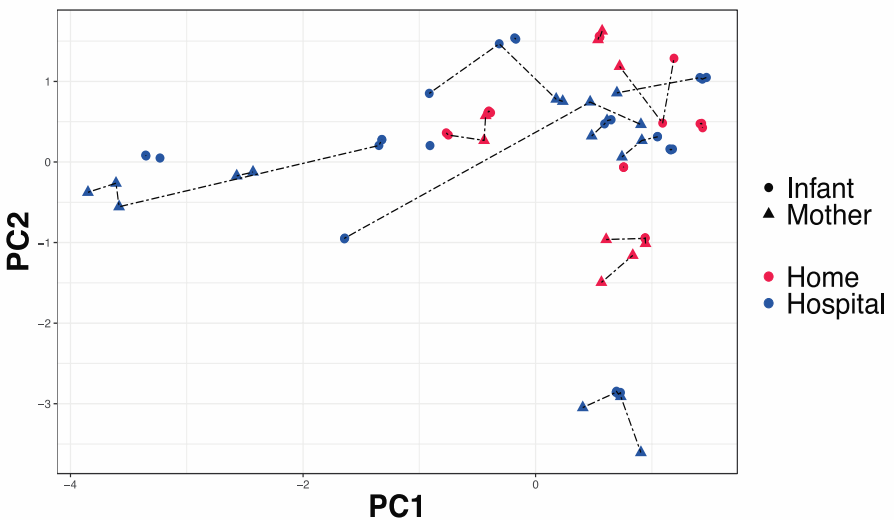

b Akkermansia muciniphila

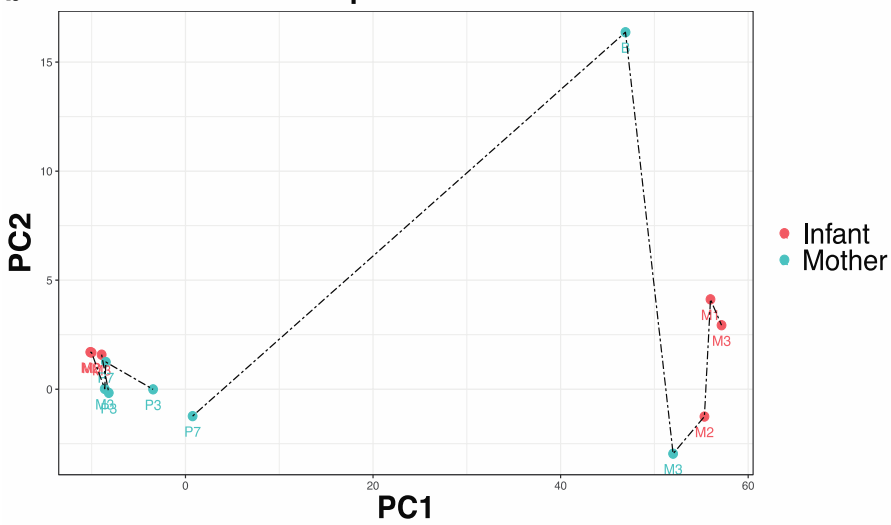

c

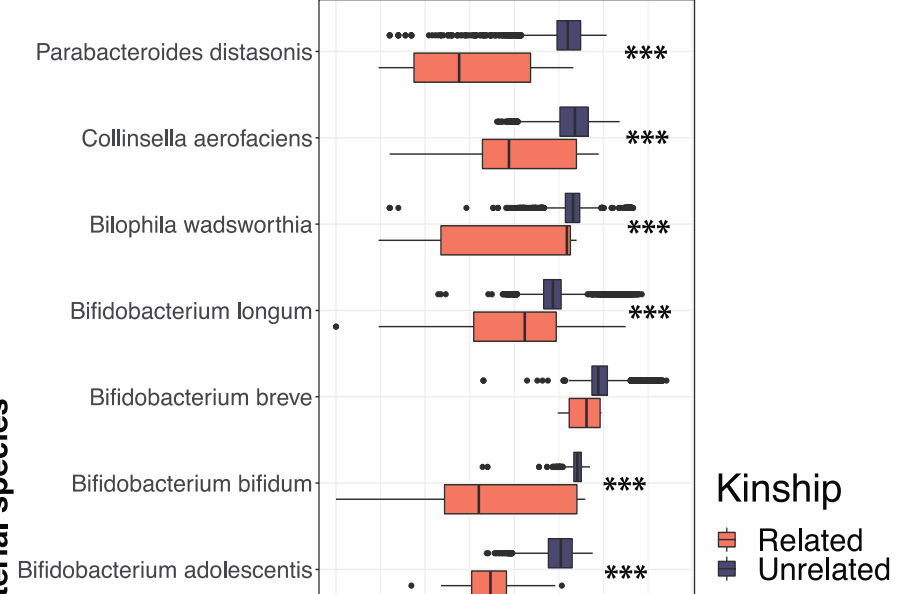

Bacteroides vulgatus

Bacteroides uniformis

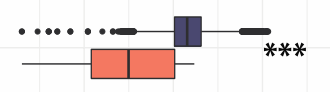

Bacteroides uniformis

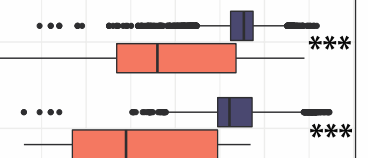

Bacteroides dorei-

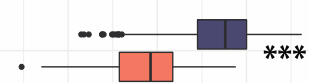

Alistipes finegoldii

Akkermansia muciniphila

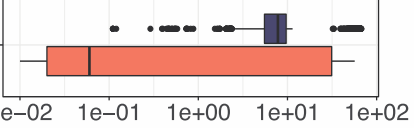

Log-scaled Kimura distance

Figure 2: Patterns of within- species phylogenetic distances between mothers and infants. 
a, pairwise distances between $B$. bifidum strains between mother-infant pairs delivered at the hospital (HS) and mother-infant pairs delivered at home (HM). b, PCoA based on phylogenetic distances in A. muciniphila strains in mother-infant pairs. Labels represent timepoints. Only strains present in at least one mother-infant pair are depicted c, Phylogenetic distances within all combinations of samples for related mothers and infants and distances between unrelated individuals.

\section{Gut viral composition in mothers and infants}

114 We performed VLP DNA extraction for 93 faecal samples from mothers (pregnancy and birth

115 timepoints) and infants (birth, month 1, month 2 and month 3 ) that were selected for total

116 metagenome analysis based on sufficiency of faecal material (Figure 1a). In all, 66 VMs were

117 successfully sequenced (Figure 1a) and 51,449 viral-representative contigs were

118 reconstructed from all successfully sequenced VMs, constituting a curated viral database

119 (Figure 3a). Of these, 616 contigs, ranging in size from 3 to $253 \mathrm{kbp}$, had identical ends, which

120 suggests that they represent complete genomes of viruses with circular or terminally

121 redundant linear genomes.

122 The total number of contigs with an assigned taxonomy at family level was 14,405

123 (28\% of all detected viral contigs). Viral-representative contigs were assigned to 11 families

124 of eukaryotic and prokaryotic viruses. Consistent with previous observations $99.7 \%$ of the

125 taxonomically assigned contigs represented bacteriophages ${ }^{12,13}$. Among the seven

126 bacteriophage families, Siphoviridae represented the largest number of contigs. Based on

127 similarity between viral contigs and CRISPR spacer sequences of microbial genomes, we

128 predicted potential hosts for 630 viral contigs (1.2\%; Supplementary Table S5; see Methods).

129 Most infant bacteriophages were linked to bacteria of the phyla Actinobacteria, Firmicutes

130 and Proteobacteria whereas maternal bacteriophages were linked to Firmicutes, 
131 Actinobacteria and Bacteroidetes and a very low fraction were linked to Proteobacteria

132 (Figure 3c).
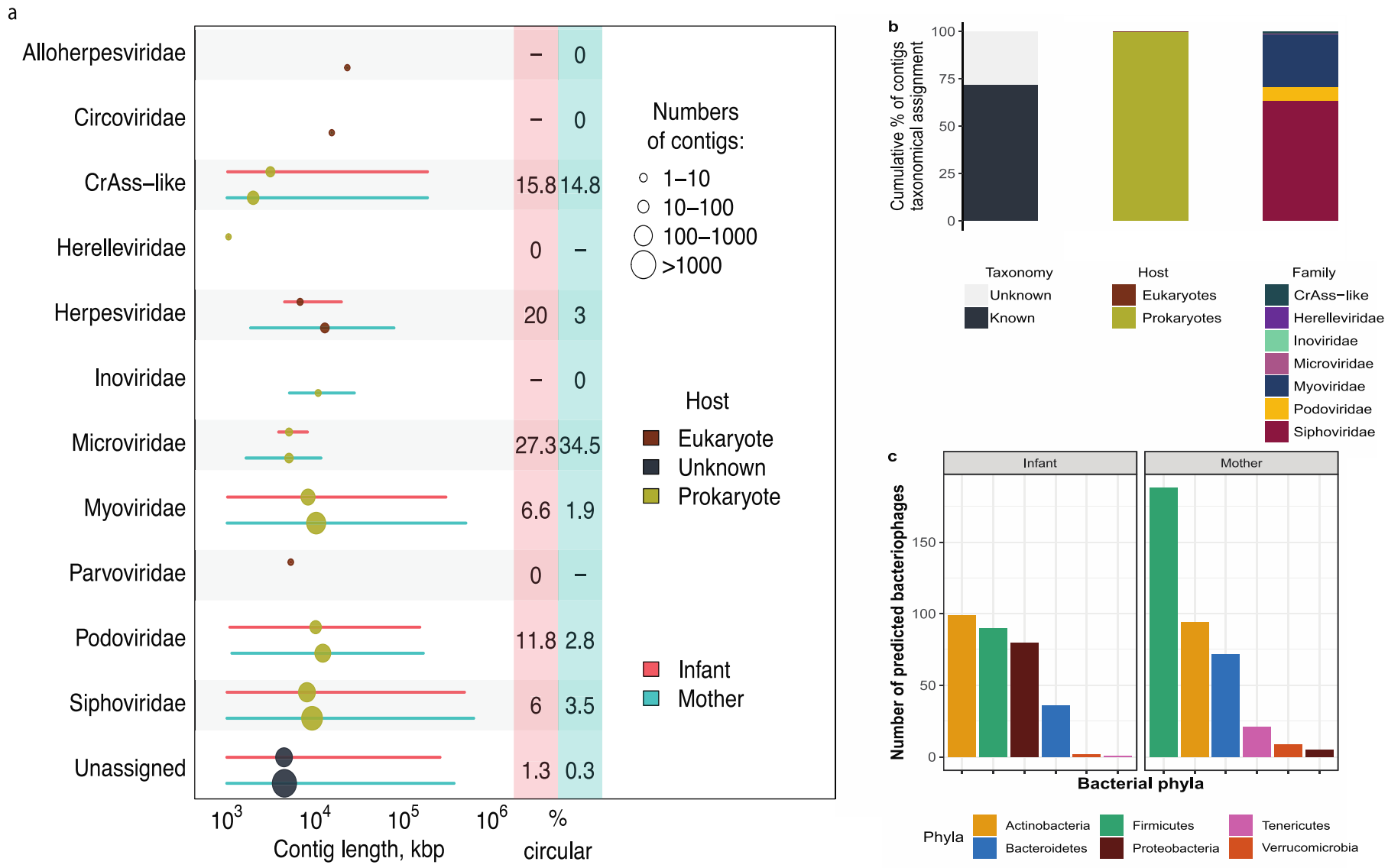

Figure 3: Characteristics of the viral representative contigs.

a, Distribution of contigs from the curated database by length, taxonomic family and bacterial host. Blue and pink lines represent the spread of contig length in mothers and infants, respectively. The colour of the dots at the median length values indicates if a family includes eukaryotic (yellow), prokaryotic (chartreuse) or unassigned (black) viruses. b, Classification of viral contigs by taxonomy, host and viral family. c, Number of representative viral contigs linked to their potential bacterial phyla by CRISPR.

133 To obtain information about the prevalence and presence of viral-representative contigs in

134 VMs, we aligned their reads to the curated viral database (see Methods). Here, we observed

135 that virome composition at viral family level was mostly consistent across maternal samples,

136 with high prevalence of viral contigs assigned to the bacteriophage families Siphoviridae, 
137 Podoviridae, Myoviridae and crAss-like phages that were present in all maternal samples

138 (Figure 4a). In maternal samples, the abundances of these most prevalent viral families did 139 not change significantly over time (FDR>0.05, Figure 4a).

140 Similar to the maternal samples, the viral families Siphoviridae, Podoviridae and 141 Myoviridae were present in all infant samples, and the crAss-like phages were present in 31 142 out of 32 infant samples. In infants, the abundances of these viral families did not change 143 significantly with time. While abundances of the Podoviridae and Myoviridae viral families 144 were similar between maternal and infant samples, the abundance of crAss-like phages was 145 significantly higher in maternal samples compared to infant samples (FDR<0.05) and the 146 abundance of the Siphoviridae family was higher in infants compared to mothers (FDR $<0.05$,

147 Figure 4a). Siphoviridae dominated both maternal and infant viromes, showing a greater 148 abundance variation and a nominally significant decrease with time in infants ( $p$-value=0.04, $149 \quad F D R=0.17)$.

150 At the level of viral-representative contigs, the composition and diversity of maternal 151 and infant samples showed remarkable differences. The alpha-diversity of the maternal 152 virome was significantly higher than that of the infant virome ( $p$-value $=2.2 e-05$, Figure $4 b$ ) 153 and did not change over time in mothers or infants. Similar to the bacterial community, we 154 observed divergent faecal viral communities between mothers and infants, with maternal and 155 infant samples forming two distinct clusters (PERMANOVA, $p$-value=0.001, 999 permutations, 156 Figure 4c), although this clustering was not as distinct as for the bacteriome composition 157 (beta-diversity R2 0.62 for bacteriome versus 0.37 for virome). Even though infant samples 158 tended to cluster together in the first principal component (Figure 4c), infant viromes were 159 more individual-specific than maternal viromes (PERMANOVA, p-value<0.001, 999 160 permutations, Figure 4d). Infant viromes also showed greater changes with time than 
162 for infants and $0.56 \pm 0.14$ for mothers) (Figure $4 d$ ), indicating more dynamic viromes in the 163 developing gut ecosystems.
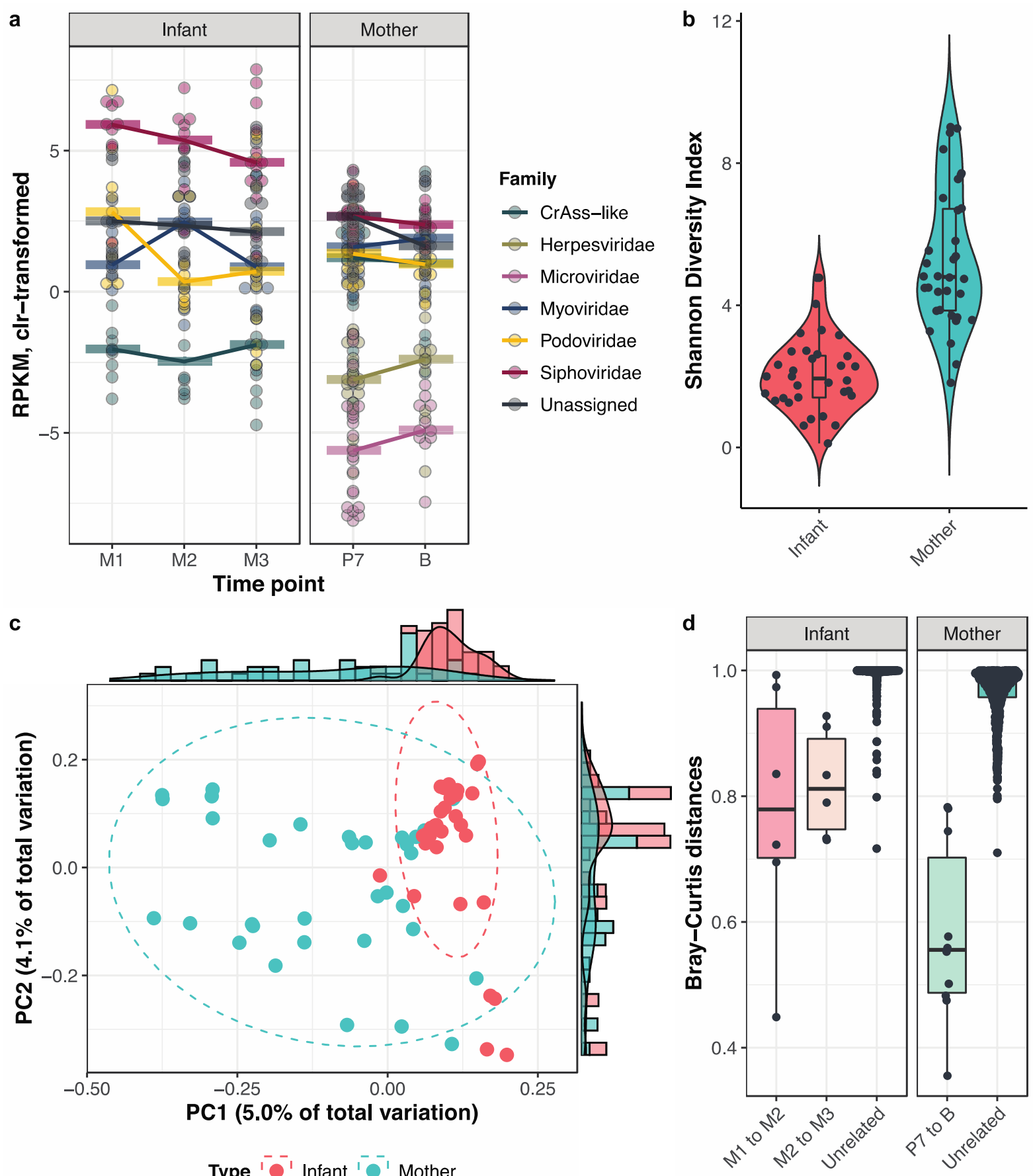

Figure 4: Differences in viral composition between mothers and infants.

a, Dynamics of viral families in mothers and infants. Reads were associated with viral lineages based on the annotation of viral contigs. Only viral families present in at least five samples per timepoint 
are shown. b, Difference in alpha-diversity (calculated using the Shannon diversity index) of viralrepresentative contigs in mothers and infants. c, PCoA based on the beta-diversity of the viruses in viral metagenomes (VMs) calculated using Bray-Curtis dissimilarity matrix. Ellipses show 95\% confidence intervals. d, Between-individual and within-individual Bray-Curtis distances for infants and mothers.

164 In mothers, 105 viral contigs were shared across at least $50 \%$ of the samples, which is in 165 contrast to only two viral contigs shared across at least $50 \%$ of the infant samples, 166 demonstrating, yet again, the high individual-specificity of the infant gut virome.

167 In mothers, the number of shared viruses across two timepoints was, on average, 168 1,060, which comprised $26.0 \pm 18.6 \%$ of all viruses ever detected in a mother (Supplementary

169 Fig.3). This percentage is comparable with another longitudinal study in adults, where $17019.4 \pm 13.5 \%$ of the total viral pool was shared between two timepoints ${ }^{13}$. In infants, on 171 average, 41 viruses, or $20.9 \pm 14.9 \%$ of all viruses ever detected in an infant, were shared across

172 two timepoints. These results indicate that the percentage of shared viruses across 173 timepoints in an individual is the same for infants and mothers, even though the viral richness 174 in infants was substantially lower.

\section{Mother to infant transmission of viruses}

175 A moderate proportion of all the viruses found in infants was shared with their mothers

$176(11.3 \pm 20.0 \%)$, which is in contrast to the proportion of bacterial species shared between

177 mothers and infants $(43.6 \pm 10.0 \%)$. The proportion of shared viruses did not significantly

178 correlate to any available infant phenotypes.

179 Previous studies have shown that the adult gut ecosystem observes "Piggyback-the-

180 Winner" dynamics, a state where bacteriophage lysogeny is increasingly favoured in a state

181 with a higher microbial density ${ }^{11,14,15}$. In contrast, the developing infant gut ecosystem is 182 thought to be characterised by a high frequency of bacterial lysis events induced by 
bacteriophages ${ }^{16}$. We therefore sought to compare the cumulative relative abundance of

184 temperate phages between mothers and infants using the presence of integrase and

185 recombinase genes within each viral contig as a measure of temperate phages. The relative

186 abundance of active temperate phages in infants was significantly higher than in mothers ( $p$ -

187 value $=7.2 e-06$, Figure $5 a)$ and decreased with timepoint $\left(R_{\text {Pearson }}=-0.38, p\right.$-value $=0.03$, Figure

188 5b). The difference in the abundances of temperate phages in infant and maternal samples

189 may explain the low proportion of shared viruses in mother-infant pairs, as our VLP extraction

190 method targets actively reproducing viruses and not prophages. We therefore investigated

191 whether the proportion of shared viruses increased when accounting for prophages

192 integrated in bacterial genomes in maternal TMs. To do so, we mapped reads from the TMs

193 to the curated viral database and obtained the relative abundances of bacteriophages found

194 in TMs. The viral richness of both mothers and infants was higher in TMs than in VMs, with $462 \pm 2767.4$ and $1508.5 \pm 2968.3$ viruses per sample for VMs and TMs, respectively ( $p$ -

196 value=3.0e-4). This was presumably due to the presence of prophages in TMs that are not

197 captured as part of the active fraction in the VMs. As in VMs, in TMs, the viral alpha-diversity

198 of maternal samples was higher than those of infants ( $p$-value<2e-16), and the samples from

199 mothers and infants formed two distinct clusters based on Bray-Curtis dissimilarity

200 (PERMANOVA, p-value<0.001, 999 permutations, Supplementary figure 4a). The composition

201 of the virome was highly consistent between concurrent VMs and TMs (Supplementary figure

202 4b). Interestingly, the proportion of viruses shared between mothers and their infants in TMs

203 was $37.6 \pm 22.5 \%$ (Figure $5 c$ ), which is much higher than in VMs (on average $27.8 \%$ higher, $p$ -

204 value=7.6e-06). The proportion of shared viruses between mothers and infants is thus similar

205 to the proportion of the shared bacterial species when the prophages are taken into account.

206 There was no significant correlation between the proportion of shared bacterial species in 
207 mother-infant pairs and the proportion of shared viruses in VMs (Figure $5 \mathrm{~d}$ ). The proportion

208 of shared bacterial species correlates moderately with the proportion of shared viruses in

209 TMs (Rearson $=0.47, p=0.006$, Figure 5e), further suggesting that vertically transmitted bacteria

210 could be a source for vertically transmitted bacteriophages. These findings together support

211 the argument that the phages colonising the infant gut are vertically transmitted from the

212 mother by means of vertically transmitted bacteria. 

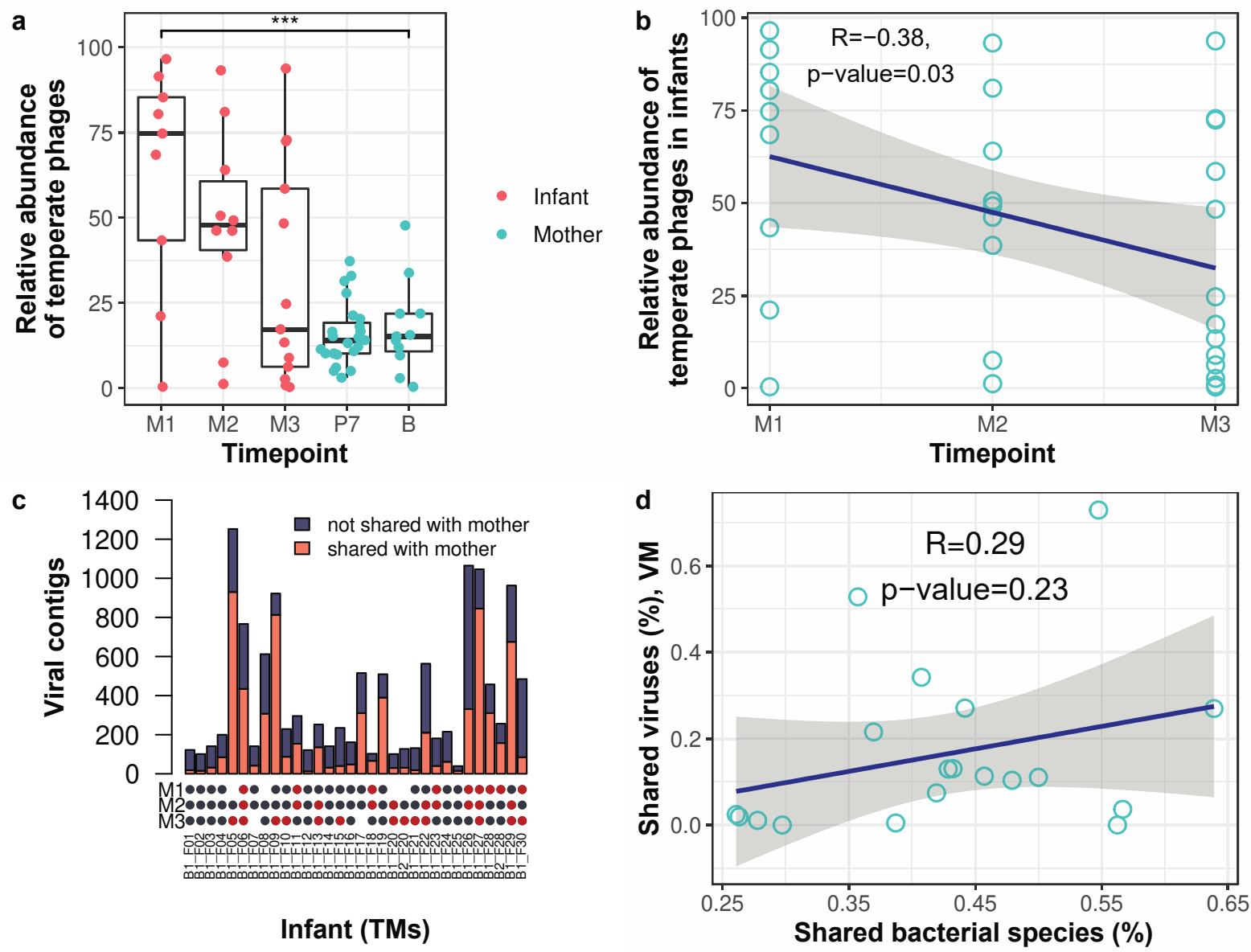

Infant (TMs)

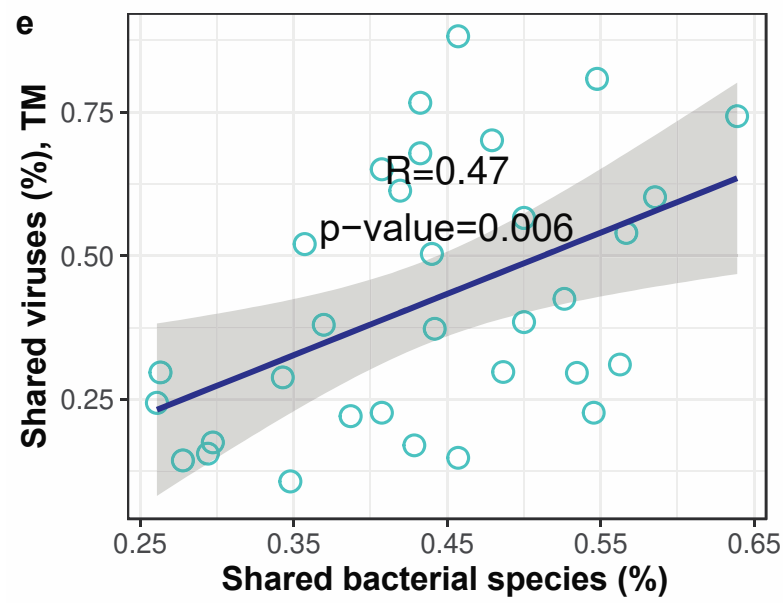

\section{Figure 5: Maternal to infant transmission of viruses}

a, Relative abundance of temperate phages in mothers (P7, B) and infants (M1, M2, M3). b, The relative abundance of temperate phages in infants decreasing with time. $c$, The number of the shared viral-representative contigs between related and unrelated infants and mothers in total metagenome (TM) samples. Number of dots represents the number of timepoints available for each infant and the red dots represent the availability of VM's for that sample $d$, Correlation of the proportion of shared bacterial and the proportion of shared viruses in VMs of mother-infant pairs. e, 
Correlation of the proportion of shared bacterial species and the proportion of shared viruses in TMs of mother-infant pairs.

\section{Discussion}

213 This study describes the extent and nature of microbial transmission from mothers to infants,

214 with a focus on viral transmission. A limitation of our study design is that we did not analyse

215 RNA viruses, given their perceived low abundance in the healthy human gut, although future

216 studies should also investigate this dark aspect of the virome. However, while we

217 acknowledge that our sample size only allows us to describe a limited number of associations

218 with phenotypes and only indicates trends in the co-development of bacteriome and virome,

219 our study also has notable strengths. Our novel approach of complementary investigation of

220 both TMs and VMs in both mothers and infants led to novel findings. In the VLP-specific

221 analysis that selectively explores the active virome fraction in stool, we observe high

222 individual-specificity and lower diversity in infants compared to mothers. The average shared

223 fraction of active virome between infants and their mothers was $11.3 \%$, which agrees with

224 the results of one of the few studies to investigate the sharedness of viruses amongst mothers

225 and their infants, where a low sharing of viruses in comparison with bacterial genera was

226 reported in 28 mother-infant twin pairs ${ }^{16}$. However, in our study, when we took prophages

227 into account, the average proportion of vertically transmitted viruses increased to $37.6 \%$ and

228 became comparable to the sharedness of gut bacterial species. The majority of the temperate

229 bacteriophages dominating the infant gut were not detected in maternal metaviromes, but

230 were prevalent in maternal TMs, suggesting that infants partly obtain their virome from their

231 mothers via vertically transmitted bacteria.

232 Our study also addressed previously unstudied factors for maternal to infant microbial

233 transmission such as the place of deliveries. As home deliveries constitute $12.7 \%$ of the total 
234 deliveries (2018) in the Netherlands, and $28 \%$ in our samples, we possess the unique 235 opportunity to also reveal trends concerning possible novel associations regarding the effect 236 of place of birth on infant bacterial strains like Bifidobacterium bifidum which need to be

237 further investigated in a larger cohort ${ }^{17}$. An additional strength of our study was that we did 238 not use amplification techniques during the generation of VMs, which allowed accurate 239 quantification of viruses and led to minimal bias in our estimation and characterisation of 240 double stranded DNA viral families.

241 In conclusion, we provide evidence for mother-to-child viral and bacterial

242 transmission events at high-resolution and novel insights into the early colonisation of the 243 infant gut ecosystem. 


\section{Methods}

\section{Study design}

244 The samples for this (pilot) study were obtained from the Lifelines NEXT cohort, a birth cohort designed to study the effects of intrinsic and extrinsic determinants on health and disease in

246 a four-generation design. Lifelines NEXT is embedded within the Lifelines cohort study, a

247 prospective three-generation population-based cohort study recording the health and healthrelated aspects of 167,729 individuals living in Northern Netherlands ${ }^{18}$ In Lifelines NEXT, we aim to include 1,500 pregnant Lifelines participants and intensively follow them, their partners and their children up to at least 1 year after birth. During the Lifelines NEXT study, biomaterials including maternal and neonatal (cord) blood, placental tissue, faeces, breast milk, nasal swabs and urine are collected from the mother and child at 10 timepoints. The long-term health outcomes of these infants and their parents will be investigated. Furthermore, data on medical, social, lifestyle and environmental factors are collected via questionnaires at 14 different timepoints and via connected devices ${ }^{19}$.

\section{Informed consent}

256 The Lifelines NEXT study was approved by the Ethics Committee of the University Medical

257 Center Groningen, document number METC UMCG METc2015/600. Written informed consent forms were signed by the participants or their parents/legal guardians.

\section{Sample collection}

Mothers collected their faeces during pregnancy at months 3 and 7, at birth and 3 months after birth (Figure 1a). Faeces from infants were collected from diapers by their parents at months 1, 2 and 3 of infant age. Parents were asked to freeze the stool samples at home at $20^{\circ} \mathrm{C}$ within $10 \mathrm{~min}$ of stool production. Frozen samples were then collected and transported to the UMCG and stored in a $-80^{\circ} \mathrm{C}$ freezer until extraction of microbial and viral DNA. We collected a total of 217 samples for total microbiome analysis.

\section{Total microbial DNA extraction}

Microbial DNA was isolated using the QIAamp Fast DNA Stool Mini Kit (Qiagen, Germany) using the QIAcube (Qiagen) from 0.2-0.5 g faecal material, with a final elution volume of 100 $\mu \mathrm{l}$. DNA eluates were stored at $-20^{\circ} \mathrm{C}$. 


\section{VLP extraction and nucleic acid extraction}

Initially, a subset of 93 of 217 faecal samples was selected for viral -like particle extraction based on the availability of faecal material. This subset of 93 samples included maternal samples from pregnancy and birth and infant samples at birth, month 1, month 2 and month 3. The gut virome was studied using extraction of DNA from VLPS as described in Shkoporov et al. $2018^{20}$. Briefly, $0.5 \mathrm{~g}$ of faecal material was resuspended in $10 \mathrm{ml}$ of SM buffer. Samples were centrifuged at $4,800 \mathrm{rcf}$ for $10 \mathrm{~min}$ at $4^{\circ} \mathrm{C}$. Supernatant was collected and centrifuged again using the same settings. The supernatant was filtered twice through a $0.45-\mu \mathrm{m}$ pore polyethersulfone membrane filter. VLPs were concentrated from the filtrate with Polyethylene glycol 8000 (Sigma-Aldrich, Cat\#P2139) precipitation overnight and purified with chloroform treatment. The resulting fraction was treated with $8 \mathrm{U}$ of TURBO DNase (Ambion/ThermoFisher Scientific) and $20 \mathrm{U}$ of RNase I (ThermoFisher Scientific) at $37^{\circ} \mathrm{C}$ for 1 $\mathrm{h}$ before inactivating enzymes at $70^{\circ} \mathrm{C}$ for $10 \mathrm{~min}$. Subsequently, proteinase $\mathrm{K}$ ( $40 \mu \mathrm{g}$, SigmaAldrich, Cat\#2308) and $20 \mu \mathrm{l}$ of $10 \%$ SDS were added to the samples and incubated for $20 \mathrm{~min}$ at $56^{\circ} \mathrm{C}$. Finally, VLPs were lysed by addition of $100 \mu$ of Phage Lysis Buffer (4.5 M guanidinium isothiocyanate, $44 \mathrm{mM}$ sodium citrate $\mathrm{pH} 7.0,0.88 \%$ sarkosyl, $0.72 \%$ 2-mercaptoethanol) and incubated at $65^{\circ} \mathrm{C}$ for $10 \mathrm{~min}$. Nucleic acids were extracted twice from lysates using Phenol/Chloroform/Isoamyl Alcohol 25:24:1 (ThermoFisher Scientific) treatment followed by centrifugation at $8,000 \mathrm{~g}$ for $5 \mathrm{~min}$ at room temperature. The resulting aqueous phase was subjected to the final round of purification using the DNeasy Blood \& Tissue Kit (Qiagen) with a final elution volume of $50 \mu \mathrm{l}$. Viral DNA was stored at $-20^{\circ} \mathrm{C}$.

\section{Genomic library preparation and sequencing}

288 Faecal microbial DNA and viral DNA samples were sent to Novogene, China for genomic 289 library preparation and shotgun metagenomics sequencing. Sequencing libraries were 290 prepared using the NEBNext ${ }^{\circledR}$ Ultra ${ }^{\mathrm{TM}}$ DNA Library Prep Kit or the NEBNext ${ }^{\circledR}$ Ultra $^{\mathrm{TM}}$ II DNA

291 Library Prep Kit, depending on the sample DNA concentration, and sequenced using HiSeq 2000 sequencing with $2 \times 150$ bp paired-end chemistry (Illumina). On average, 30.76 \pm 4.2

293 million paired-end total metagenome reads and $29.8 \pm 4.8$ million paired-end viral metagenome reads were generated per sample. Of 217 collected samples for total microbiome analysis, 187 were successfully sequenced. Following this, three meconium samples and one mislabelled sample were excluded. For virome analysis, 66 of the 93 samples 
were successfully sequenced. As with bacteriome, meconium samples failed sequencing due

298 to their perceived low viral abundance.

\section{Profiling of total gut microbiome composition}

299 Total metagenome sequencing reads were trimmed and Illumina sequence adaptor 300 sequences removed using KneadData tools (v0.7.4) and an average PHRED quality score of $30133^{21}$. Following trimming, the KneadData-integrated Bowtie2 tool (v2.4.2) ${ }^{22}$ was used to 302 remove reads that aligned to the human genome (GRCh37/hg19), and the quality of the 303 processed data was examined using the FastQC toolkit (v0.11.9) ${ }^{23}$. Samples with a clean read304 depth $<5$ million were not considered in further analysis. Taxonomic composition of total 305 metagenomes was profiled using the MetaPhIAn3 tool with the MetaPhIAn database of 306 marker genes mpa_v30 and the ChocoPhIAn pan-genome database (201901).

\section{Profiling of gut virome composition}

307 VM sequencing reads were subject to quality trimming. Read mapping to the human 308 (GRCh38.p12) reference genome was performed with KneadData (v0.5.1) ${ }^{21}$. On average, $30922.1 \pm 4.5$ million paired-end VLP reads passed quality control. The quality of the raw and clean 310 reads was visualised with FastQC (v0.11.7) ${ }^{23}$. Bacterial contamination of VMs was assessed

311 by aligning reads to the single copy chaperonin gene cpn60 database ${ }^{20}$. On average, VLP samples contained $3.7 \pm 7.9 \%$ of bacterial genomic DNA per sample.

313 We annotated the gut virome composition using the de novo assembly-based 314 method. Whole metagenome de novo assembly was performed per VMs using SPAdes 315 (v3.11.1) in metagenomic mode (-meta) with default settings ${ }^{24}$. On average, 280,363 contigs 316 were assembled for maternal samples and 46,830 contigs were assembled for infant samples. 317 When pooled, 724,612 contigs from the whole dataset were subjected to a redundancyremoval procedure in which contigs with $90 \%$ nucleotide identity over $90 \%$ of the length of a 319 shorter contig were considered redundant, and the shorter contig was removed. Overall, 320274,870 representative pooled contigs larger than $1 \mathrm{kbp}$ were subject to validation as viral.

321 For the 274,870-representative pooled contigs, we predicted the Open Reading 322 Frames (ORFs) using Prodigal v2.6.3 in metagenomic mode ${ }^{25}$. A Hidden Markov Model 323 (HMM) algorithm (hmmsearch from HMMER v3.2.1 package) ${ }^{26}$ was used to compare amino 324 acid sequences of predicted protein products against the HMM database Prokaryotic Virus 
325 Orthologous Groups (pVOGs) ${ }^{27}$. Hits were considered significant at an e-value threshold of

$32610^{-5}$. Ribosomal proteins were identified using a BLASTp ${ }^{28}$ search (e-value threshold of $10^{-10}$ )

327 against a subset of ribosomal protein sequences from the COG database (release 2014). We used VirSorter v1.0.3 ${ }^{29}$ with its expanded built-in database of viral sequences ('-db 2' parameter) in the decontamination mode as one of the steps for prediction of viral sequences. Representative contigs larger than $1 \mathrm{kbp}$ were considered viral if they fulfilled at least one of six criteria (similar to those described in ${ }^{12,13,30}$ ): (1) they produced BLASTn alignments to a

332 viral section of NCBI RefSeq (release 98) with e-value $\leq 10^{-10}$, covering $>90 \%$ of contig length at $>50 \%$ identity, (2) they had at least three ORFs, producing HMM-hits to the pVOG database with an e-value $\leq 10^{-5}$, with at least two ORFs per $10 \mathrm{~kb}$ of contig length, (3) they were VirSorter-positive (all six categories, including suggestive), (4) they were circular ${ }^{31},(5)$ they produced BLASTn alignments to 427 crAss-like reference genomes ${ }^{32} \&$ unpublished data) with an e-value $\leq 10^{-10}$ covering $>90 \%$ of contig length at $>50 \%$ identity, or (6) they were longer than $3 \mathrm{kbp}$ with no hits to the nt database (release 235) (alignments $>100$ nucleotides with $90 \%$ identity and an e-value of $\left.10^{-10}\right) \cdot 54,267$ contigs fulfilled at least one of these six criteria, and these contigs were subjected to clustering with 427 crAss-like phage contigs from ${ }^{32}$ and an unpublished custom database and genomes of the reference database "ProkaryoticViralRefSeq97-Merged", using vConTACT2 0.9.15 with default parameters ${ }^{33}$.

343 Contigs assigned the status 'Overlap', 'Singleton' and 'Outlier' by vConTACT2 were treated as

344 viral clusters consisting of a single contig in all subsequent analyses. Contigs were further subject to a decontamination procedure based on the following criteria. Contigs were kept if they 1) were circular and contained at least 1 pVOGs hit, 2) were circular and VirSorterpositive, or 3) were VirSorter-positive and did not have ribosomal protein genes. Contigs were excluded if they 1) had more than three ribosomal protein genes (similar to the decontamination process described in $\left.{ }^{12,13}\right)$, or 2) were longer than $200 \mathrm{kbp}$ and co-clustered with contigs possessing more than three ribosomal protein genes. dataset included 51,455 representative contigs ranging in size from $1 \mathrm{kbp}$ to $>626 \mathrm{kbp}$, with low-to-high k-mer coverage $(1.1-52,101.3 \mathrm{X})$, which recruited $89 \%$ reads per sample on average. 
35651,455 viral-representative contigs on a per-sample basis using Bowtie2 v2.3.4.1 in 'end-to-

357 end' mode ${ }^{22}$ A count table was subsequently generated using SAMTools v1.9 ${ }^{34}$. Sequence coverage was calculated per contig per sample using the BEDtools v2.25.0 'coverage' command ${ }^{35}$. To remove spurious Bowtie 2 alignments, readcounts that featured a breadth of contig coverage less than $1 \times 75 \%$ of a contig length were set to zero ${ }^{36}$ resulting in 51,449 viral sequences being used for the construction of the final count table. RPKM value transformation was applied to the final count table, and the resulting RPKM count table was used for downstream analysis.

Family-level taxonomic annotations were assigned to viral contigs using the Demovir script (https://github.com/feargalr/Demovir) with default parameters and database. Manual curation of Demovir taxonomy assignment and expansion on unassigned contigs using vConTACT was performed as described previously ${ }^{13}$. Despite using sequencing adaptors binding only dsDNA, a few genomes of ssDNA viruses assigned to the families Circoviridae, Inoviridae, Microviridae and Parvoviridae were reconstructed. These could be either a result of taxonomy misassignment or of catching these viruses during their replication in the duplex form. Given the low bacterial contamination level, we did not exclude the putatively ssDNA virus contigs from the further analysis, assuming that even in case of taxonomy misassignment, these contigs are still of viral origin.

\section{Virome annotation in TMs}

374 Quality-filtered reads from 183 TMs were aligned to the custom viral database consisting of 51,455 viral-representative contigs on a per-sample basis using Bowtie2 v2.3.4.1 in 'end-toend' mode ${ }^{22} \mathrm{~A}$ count table was generated and transformed as with VMs. The resulting RPKM count table was used to assess the number of shared viruses in related mother-infant pairs. The increase in the number of shared viruses in mother-infant pairs in TMs compared to VMs did not depend on the initial size of the virus pool or any of the infant or maternal phenotypes studied (Spearman correlation, $\mathrm{p}$-value>0.05). All comparisons regarding the VMs and TMs viromes were performed on the 66 samples that had both VM and TM samples.

\section{Prediction of virus hosts based on CRISPR spacer sequences} the 51,455 viral contigs using BLASTN 2.7.1+ ${ }^{28}$ with the "-task 'blastn-short' -evalue 1e-5" 
parameters. Hosts were predicted based on hits characterised by bitscore $\geq 45$ and $<2$ mismatches, gaps and unaligned spacer nucleotides. Host taxonomy was retrieved from GenBank using BioPerl 1.6.924 ${ }^{38,39}$.

\section{Ecological measurements and taxonomic comparisons}

387 To assess bacterial alpha-diversity for each sample, we used species reported in the MetaPhIAn3 output at $>0.1 \%$ mean relative abundance and present in at least two samples. For the virome alpha-diversity measurements, no filters were applied to the table of RPKM (reads per kilobase per million) counts. The alpha diversity for both bacteriome and virome was calculated using the Shannon diversity index using the diversity () function in R package 'vegan' v.2.5-7 ${ }^{40}$. To test the difference in Shannon diversity index between mother and infant and the effect of timepoint on infant Shannon diversity index, we used a linear model or a linear mixed model, by comparing the suitability of the random effects using the exactLRT() function from the 'RLRsim' package ${ }^{41}$.

To compare the bacterial phylum abundances of the five most abundant phyla of mothers and infants, we used a linear model or a linear mixed model, by comparing the suitability of the random effects using the exactLRT() function from the ' $R L R s i{ }^{\prime}$ ' package ${ }^{41}$. Prior to analysis, a centred log-ratio (CLR) transformation was performed. FDR correction was applied to correct for multiple testing, with changes considered statistically significant at FDR $<0.05$ using the using the Benjamini-Hochberg method.

The Bray-Curtis distances between samples were calculated based on the level of bacterial species and on viral representative contigs. For the Bray-Curtis distances calculation, we used the function vegdist() from the R package 'vegan' v.2.5-740. To compare the bacterial and viral composition between mothers and infants, PERMANOVA was used with 999 permutations. For these comparisons the aforementioned filters for abundance and presence of bacterial species were used. For the viral-representative contigs-level no filters were applied.

409 The dynamics of viral family abundances was only assessed families present in at least five

410 individuals per every timepoint ( $n=4$; crAss-like phages, Myoviridae, Podoviridae and 411 Siphoviridae). To normalise the RPKM counts per sample for these viral families, we used a clr 412 transformation. We further excluded outliers if they were not in the range [Q1 - 1.5*IQR; Q3 $413+1.15^{*}[\mathrm{QR}]$, where Q1 and Q3 are the $25^{\text {th }}$ and $75^{\text {th }}$ percentiles and IQR is the interquartile 
414 range. Next, to determine if the abundance of the viral families changed over time, we applied

415 a linear (mixed) model that took into account a timepoint (and the repeated measurements).

416 Differentially abundant viral taxa between adults and infants were defined using a linear

417 (mixed) model that took into account the origin of the sample (and the repeated

418 measurements). The choice of model for every viral family was performed by comparing the

419 suitability of the random effects, as described earlier. FDR correction was applied to correct

420 for multiple testing as described previously.

\section{Bacterial species-specific strain analysis}

422 Strain SNP haplotypes were generated using StrainPhIAn3 ${ }^{21,42}$. This method is based on

423 reconstructing consensus sequence variants within species-specific marker genes and using 424 them to estimate strain-level phylogenies ${ }^{42}$. This method only takes into account the 425 dominant strain of species and hence our method misses overlaps in secondary strains. We 426 then performed multiple sequence alignment and used the Kimura 2-parameter method from 427 the 'EMBOSS' package ${ }^{43}$ to calculate phylogenetic distance matrices that contain the pairwise 428 nucleotide substitution rate between strains. Using presence in more than 50 samples as a 429 cut-off, we were able to construct SNP haplotype differences in 17 bacterial species. To 430 identify distinct strain clusters within species, we performed hierarchical clustering using the $431 \mathrm{R}$ function hcluster(). To define identical strains between mother and infant, a definition of 4320.0 of Kimura strain distance value was used. The significance of the difference between 433 phylogenetic distances between related mother-infant pairs and unrelated mother-infant 434 pairs was tested using PERMANOVA (999 permutations) and a FDR<0.05 was considered 435 significant. Similarly, the effect of place of birth was tested using PERMANOVA (999 436 permutations) on the phylogenetic distances of Bifidobacterium bifidum correcting for 437 samples origin (mother or infant) and repeated measurements.

\section{Association with phenotypes}

438 We used R package 'Multivariate Association with Linear Models (MaAsLin) 2' 44 to associate 439 phenotype data with bacterial species. MaAsLin performs boosted, additive general linear 440 models of associations between phenotypes and the relative abundance of species. We 441 performed this analysis only in infant samples where we used a cut-off of $0.01 \%$ species 442 abundance and selected only species occurring in at least $5 \%$ of the samples. Prior to input 443 into MaAsLin, we performed a clr transformation. In MaAsLin, we set individual IDs as random 
444 effects and added read-depth, sex, delivery mode, infant feeding type, timepoint, birth 445 weight, place of birth, maternal age, maternal pre-pregnancy BMI and gestational age as fixed 446 effects. We defined statistical significance at FDR<0.05. Given the low sample size to study 447 association of viral contig abundances with phenotypes we did not perform this analysis.

\section{Code availability}

448 All codes used in this study can be found at: https://github.com/GRONINGEN-MICROBIOME-

449 CENTRE/Lifelines NEXT 


\section{List of citations}

1. Fan, Y. \& Pedersen, O. Gut microbiota in human metabolic health and disease. Nat. Rev. Microbiol. 19, 55-71 (2021).

2. Robertson, R. C., Manges, A. R., Finlay, B. B. \& Prendergast, A. J. The Human Microbiome and Child Growth - First 1000 Days and Beyond. Trends Microbiol. 27, 131-147 (2019).

3. Tamburini, S., Shen, N., Wu, H. C. \& Clemente, J. C. The microbiome in early life: implications for health outcomes. Nat. Med. 22, 713-722 (2016).

4. Van Daele, E., Knol, J. \& Belzer, C. Microbial transmission from mother to child: improving infant intestinal microbiota development by identifying the obstacles. Crit. Rev. Microbiol. 45, 613-648 (2019).

5. Yassour, M. et al. Strain-Level Analysis of Mother-to-Child Bacterial Transmission during the First Few Months of Life. Cell Host Microbe 24, 146-154.e4 (2018).

6. Ferretti, P. et al. Mother-to-Infant Microbial Transmission from Different Body Sites Shapes the Developing Infant Gut Microbiome. Cell Host Microbe 24, 133-145.e5 (2018).

7. Megli, C. J. \& Coyne, C. B. Infections at the maternal-fetal interface: an overview of pathogenesis and defence. Nat. Rev. Microbiol. 1-16 (2021) doi:10.1038/s41579-021-00610-y.

8. Garmaeva, S. et al. Studying the gut virome in the metagenomic era: challenges and perspectives. BMC Biol. 17, 84 (2019).

9. Brum, J. R., Hurwitz, B. L., Schofield, O., Ducklow, H. W. \& Sullivan, M. B. Seasonal time bombs: dominant temperate viruses affect Southern Ocean microbial dynamics. ISME J. 10, 437-449 (2016).

10. Emerson, J. B. et al. Host-linked soil viral ecology along a permafrost thaw gradient. Nat. Microbiol. 3, 870-880 (2018).

11. Liang, G. et al. The stepwise assembly of the neonatal virome is modulated by breastfeeding. Nature 581, 470-474 (2020). 
12. Shkoporov, A. N. et al. The Human Gut Virome Is Highly Diverse, Stable, and Individual Specific. Cell Host Microbe 26, 527-541.e5 (2019).

13. Garmaeva, S. et al. Stability of the human gut virome and effect of gluten-free diet. Cell Rep. 35, 109132 (2021).

14. Knowles, B. et al. Lytic to temperate switching of viral communities. Nature 531, 466-470 (2016).

15. Redgwell, T. A. et al. Prophages in the infant gut are largely induced, and may be functionally relevant to their hosts. http://biorxiv.org/lookup/doi/10.1101/2021.06.25.449885 (2021) doi:10.1101/2021.06.25.449885.

16. Maqsood, R. et al. Discordant transmission of bacteria and viruses from mothers to babies at birth. Microbiome 7, 156 (2019).

17. De Staat van Volksgezondheid en Zorg. Bevallingen. https://www.staatvenz.nl/kerncijfers/bevallingen.

18. Scholtens, S. et al. Cohort Profile: LifeLines, a three-generation cohort study and biobank. Int. J. Epidemiol. 44, 1172-1180 (2015).

19. Warmink-Perdijk, W. D. B. et al. Lifelines NEXT: a prospective birth cohort adding the next generation to the three-generation Lifelines cohort study. Eur. J. Epidemiol. 35, 157-168 (2020).

20. Shkoporov, A. N. et al. Reproducible protocols for metagenomic analysis of human faecal phageomes. Microbiome 6, 68 (2018).

21. Beghini, F. et al. Integrating taxonomic, functional, and strain-level profiling of diverse microbial communities with bioBakery 3. eLife 10, e65088 (2021).

22. Langmead, B. \& Salzberg, S. L. Fast gapped-read alignment with Bowtie 2. Nat. Methods 9, 357359 (2012).

23. Wingett, S. W. \& Andrews, S. FastQ Screen: A tool for multi-genome mapping and quality control. F1000Research 7, 1338 (2018). 
24. Nurk, S., Meleshko, D., Korobeynikov, A. \& Pevzner, P. A. metaSPAdes: a new versatile metagenomic assembler. Genome Res. 27, 824-834 (2017).

25. Hyatt, D. et al. Prodigal: prokaryotic gene recognition and translation initiation site identification. BMC Bioinformatics 11, 119 (2010).

26. Eddy, S. R. Accelerated Profile HMM Searches. PLoS Comput. Biol. 7, e1002195 (2011).

27. Grazziotin, A. L., Koonin, E. V. \& Kristensen, D. M. Prokaryotic Virus Orthologous Groups (pVOGs): a resource for comparative genomics and protein family annotation. Nucleic Acids Res. 45, D491-D498 (2017).

28. Altschul, S. F., Gish, W., Miller, W., Myers, E. W. \& Lipman, D. J. Basic local alignment search tool. J. Mol. Biol. 215, 403-410 (1990).

29. Roux, S., Enault, F., Hurwitz, B. L. \& Sullivan, M. B. VirSorter: mining viral signal from microbial genomic data. PeerJ 3, e985 (2015).

30. Clooney, A. G. et al. Whole-Virome Analysis Sheds Light on Viral Dark Matter in Inflammatory Bowel Disease. Cell Host Microbe 26, 764-778.e5 (2019).

31. Crits-Christoph, A. et al. Functional interactions of archaea, bacteria and viruses in a hypersaline endolithic community: Halite metagenome. Environ. Microbiol. 18, 2064-2077 (2016).

32. Guerin, E. et al. Biology and Taxonomy of crAss-like Bacteriophages, the Most Abundant Virus in the Human Gut. Cell Host Microbe 24, 653-664.e6 (2018).

33. Bin Jang, H. et al. Taxonomic assignment of uncultivated prokaryotic virus genomes is enabled by gene-sharing networks. Nat. Biotechnol. 37, 632-639 (2019).

34. Li, H. et al. The Sequence Alignment/Map format and SAMtools. Bioinformatics 25, 2078-2079 (2009).

35. Quinlan, A. R. \& Hall, I. M. BEDTools: a flexible suite of utilities for comparing genomic features. Bioinformatics 26, 841-842 (2010). 
36. Roux, S., Emerson, J. B., Eloe-Fadrosh, E. A. \& Sullivan, M. B. Benchmarking viromics: an in silico evaluation of metagenome-enabled estimates of viral community composition and diversity. PeerJ 5, e3817 (2017).

37. Pourcel, C. et al. CRISPRCasdb a successor of CRISPRdb containing CRISPR arrays and cas genes from complete genome sequences, and tools to download and query lists of repeats and spacers. Nucleic Acids Res. gkz915 (2019) doi:10.1093/nar/gkz915.

38. Sayers, E. W. et al. GenBank. Nucleic Acids Res. 49, D92-D96 (2021).

39. Stajich, J. E. The Bioperl Toolkit: Perl Modules for the Life Sciences. Genome Res. 12, 1611-1618 (2002).

40. Jari Oksanen, M. F., Roeland Kindt, Pierre Legendre, Dan McGlinn, Peter R. Minchin, R. B. O’Hara, Gavin L. Simpson, Peter Solymos, M. Henry H. Stevens, Eduard Szoecs, Helene Wagner. vegan: Community Ecology Package. (2020).

41. Scheipl, F., Greven, S. \& Küchenhoff, H. Size and power of tests for a zero random effect variance or polynomial regression in additive and linear mixed models. Comput. Stat. Data Anal. 52, 3283-3299 (2008).

42. Truong, D. T., Tett, A., Pasolli, E., Huttenhower, C. \& Segata, N. Microbial strain-level population structure and genetic diversity from metagenomes. Genome Res. 27, 626-638 (2017).

43. Rice, P., Longden, I. \& Bleasby, A. EMBOSS: The European Molecular Biology Open Software Suite. Trends Genet. 16, 276-277 (2000).

44. Mallick, H. et al. Multivariable Association Discovery in Population-scale Meta-omics Studies. bioRxiv 2021.01.20.427420 (2021) doi:10.1101/2021.01.20.427420. 


\section{Supplementary figures:}
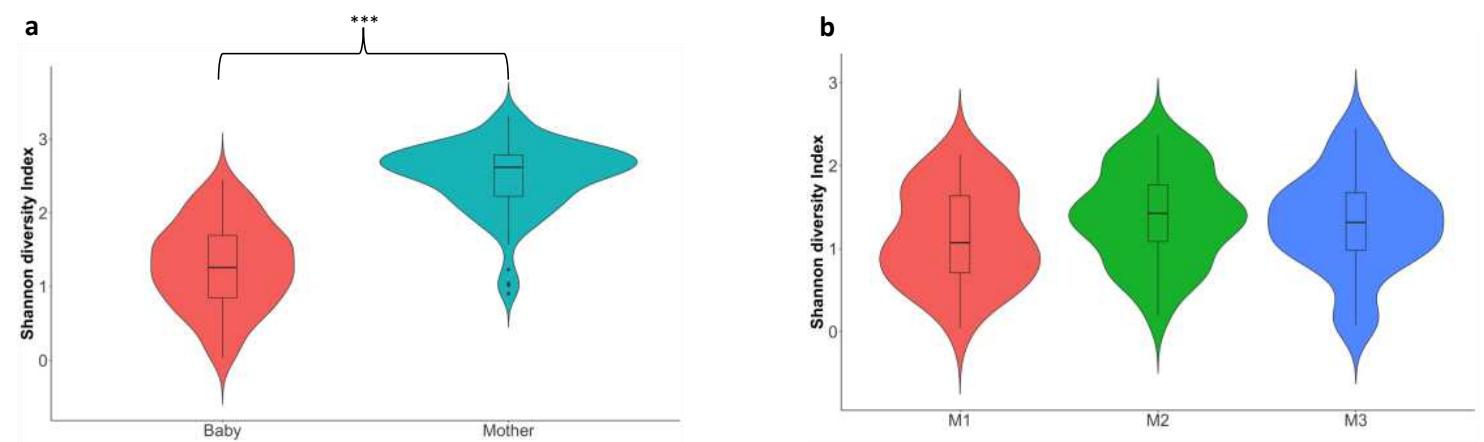

c

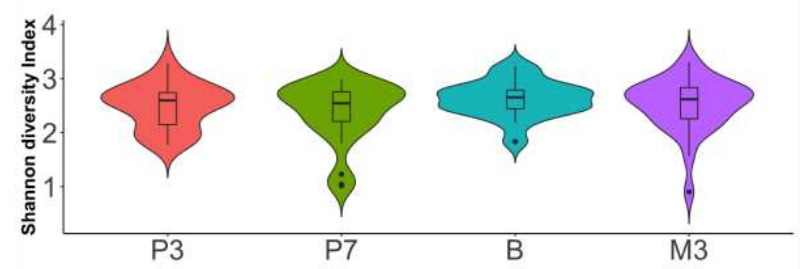

\section{Supplementary Figure 1: Violin plots representing the alpha diversity in mothers and infants}

a, Difference in alpha-diversity of bacterial species in mothers and infants $\left({ }^{* * *}\right.$ depicts a $p$ value of $<2.2 \times 10 e-16)$. Alpha-diversity of the bacteria at species level was calculated using Shannon diversity index. $\mathbf{b}$, Alpha-diversity of bacterial species in infants across timepoints. c, Alpha-diversity of bacterial species in mothers across timepoints.
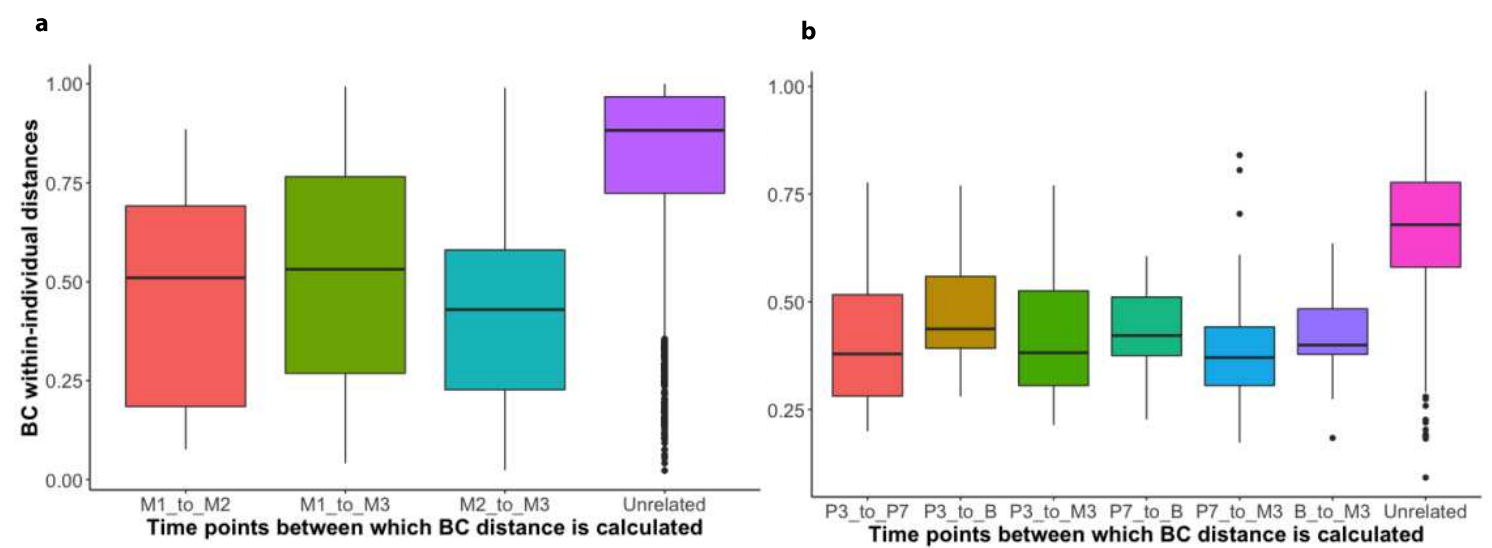

\section{Supplementary figure 2: Barplots representing beta-diversity in mothers and infants}

a, Bray-Curtis within-individual distances between timepoints in infants: Month 1 (M1) to Month 2 (M2), M1 to Month 3 (M3) and M2 to M3. The Bray-Curtis dissimilarity matrix was calculated based on the species level of bacteria. $\mathbf{b}$, Bray-Curtis within-individual distances in mothers between 
timepoints: P3-P7, P3-B, P3-M3, P7-B, P7-M3, B-M3. Bray-Curtis dissimilarity matrix was calculated based on bacterial species.

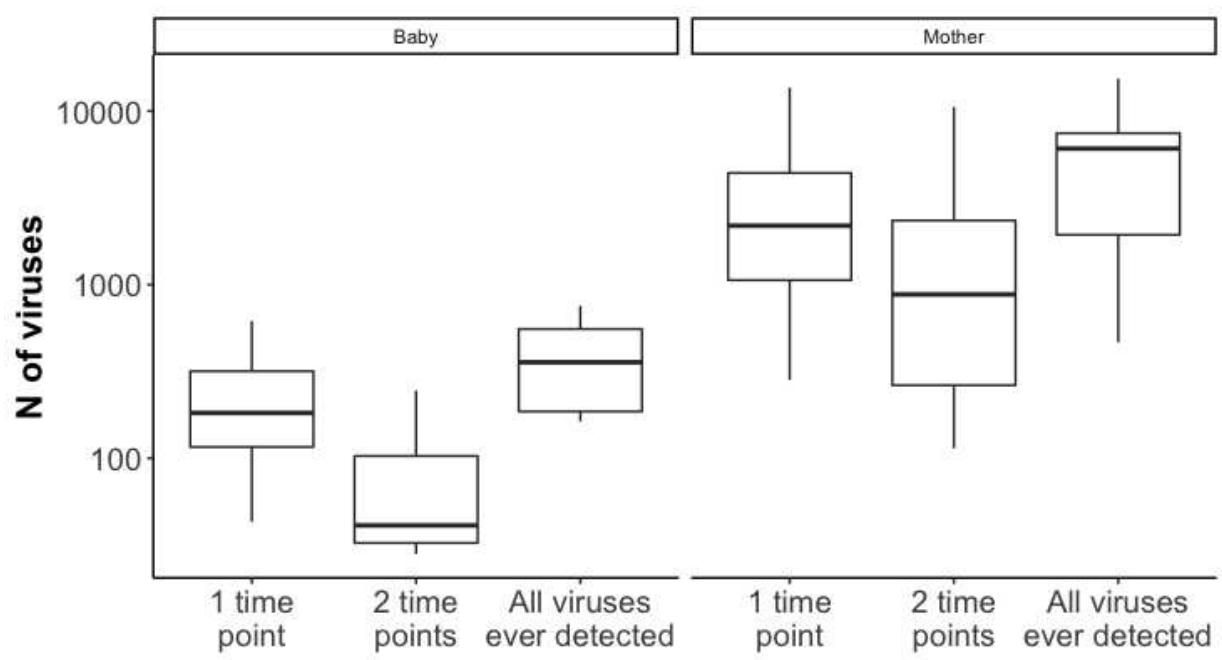

Supplementary figure 3: Number of viral-representative contigs per subject present in a given number of timepoints. Where available ( 2 infants), three timepoints were subsampled to two timepoints, and the mean was taken into consideration. Y-axis is log-scaled. "1 timepoint" is viral richness for all the infants or mothers at every timepoint. 


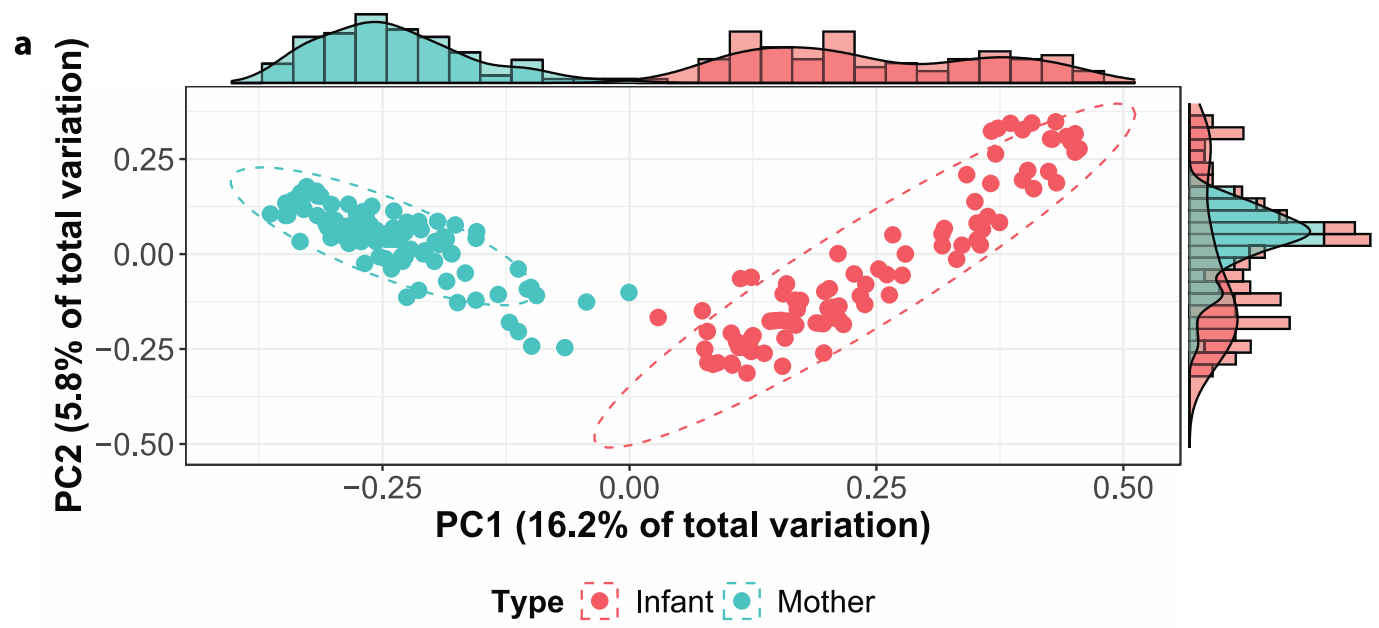

b
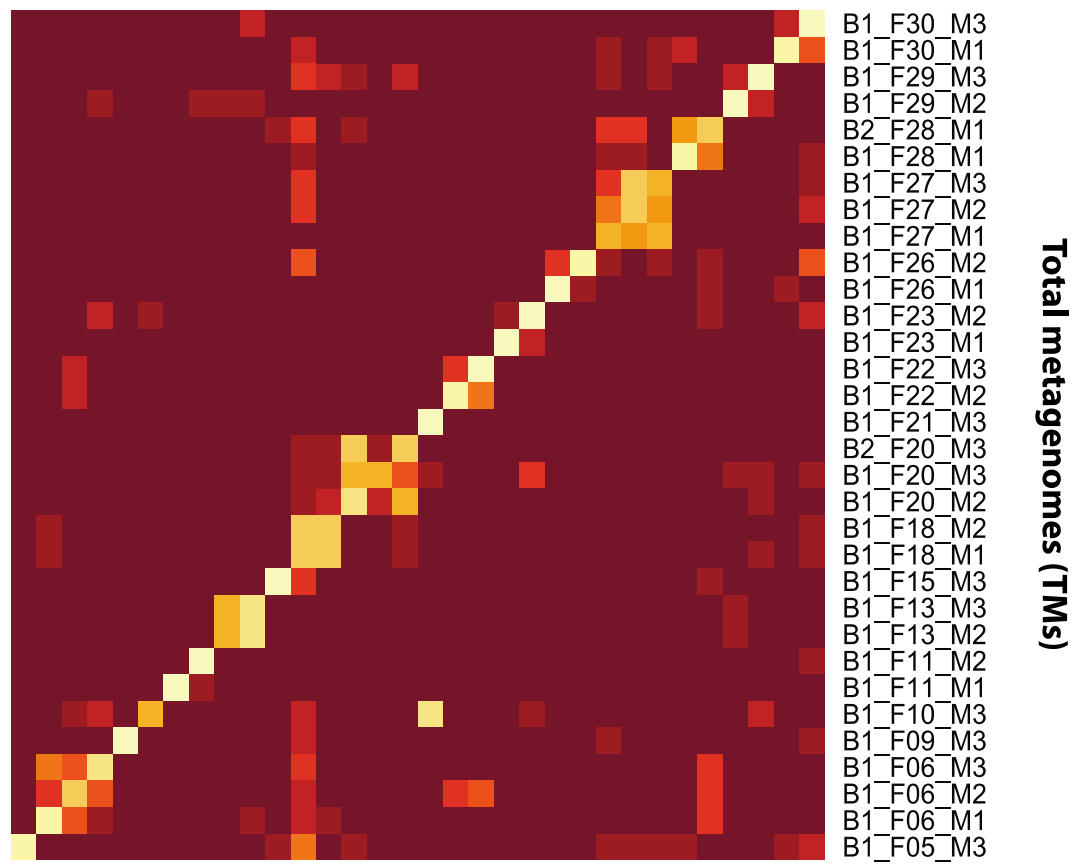

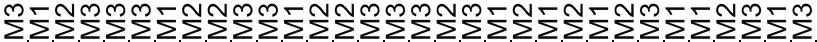

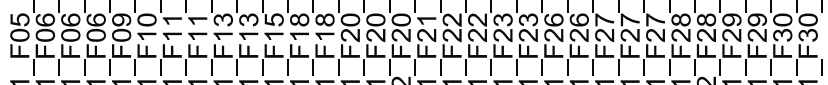

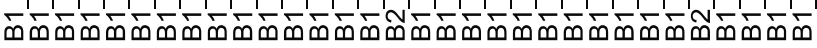

Viral metagenomes (VMs)

Supplementary figure 4: Overview of viral contigs in total metagenomes (TMs) and viral metagenomes (VMs).

a, PCoA based on the beta-diversity of the viruses in total metagenomes (TMs) calculated using BrayCurtis dissimilarity matrix. Ellipses show $95 \%$ confidence intervals. b, heatmap showing the similarity between infant individual viromes in VMs and TMs. 


\section{Acknowledgements}

We thank the participants of the Lifelines NEXT cohort for their collaboration. We thank doctors' assistants and Service bureau team of Lifelines NEXT, and significant contributions of the persons involved in the collection, processing and storage of samples: Brenda Hijmans, Annet Jansen, Gea Lamberts, Rianne de Roos, Daphne Teuben and Ettje Tigchelaar. We thank Kate Mc Intyre for editing the manuscript. We also thank the Genomics Coordination Center for providing data infrastructure and access to high performance computing clusters. We thank Daoming Wang for his assistance in the figure designs.

SG and TS hold scholarships from the Graduate School of Medical Sciences, University of Groningen and the Junior Scientific Masterclass, University of Groningen, respectively. SG was awarded a de Cocks-Hadders Stitching grant (grant number: 2021-08), and TS was awarded de Cocks Hadders Stichting grant (Winston Bakker Fonds WB-08). SB was supported by EUCAN-connect, a federated FAIR platform enabling large-scale analysis of high-value cohort data connecting Europe and Canada in personalized health. Furthermore, this project was funded by the Netherlands Heart Foundation (IN-CONTROL CVON grant 2018-27 to AZ and JF), the Netherlands Organization for Scientific Research (NWO) (NWO Gravitation ExposomeNL (024.004.017) to JF, AK and AZ, NWO-VIDI 864.13.013 and NWO-VICI VI.C.202.022 to JF, NWO-VIDI 016.178.056 to AZ and NWO Spinoza Prize SPI 92-266 to CW), the European Research Council (ERC) (ERC Advanced Grant 2012-322698 to CW, ERC Consolidator Grant 101001678 to JF and ERC Starting Grant 715772 to AZ) and the RuG Investment Agenda Grant Personalized Health to CW. JF and CW are also supported by the Netherlands Organ-on-Chip Initiative, an NWO Gravitation project (024.003.001) funded by the Ministry of Education, Culture and Science of the government of the Netherlands. The study was also financially supported with a public-private partnership allowance of Health Holland Topsector Life Sciences \& Health to stimulate public-private partnerships. The funders had no role in the study design, data collection and analysis, decision to publish, or preparation of the manuscript. 


\section{${ }^{\S}$ Lifelines NEXT cohort study}

Jackie Dekens ${ }^{1,2}$, Aafje Dotinga ${ }^{3}$, Sanne Gordijn ${ }^{4}$, Soesma Jankipersadsing ${ }^{1}$, Ank de Jonge ${ }^{5,9}$, Marlou L.A. de Kroon ${ }^{6}$, Gerard H. Koppelman7, Folkert Kuipers ${ }^{8}$, Lilian L. Peters ${ }^{5,9}$, Jelmer R. Prins $^{4}$, Sijmen A. Reijneveld ${ }^{6}$, Sicco Scherjon ${ }^{4}$, Jan Sikkema² ${ }^{2}$ Morris A. Swertz ${ }^{1}$, Henkjan J. Verkade $^{8}$, Cisca Wijmenga ${ }^{1}$, Alexandra Zhernakova ${ }^{1}$

${ }^{1}$ Department of Genetics, University of Groningen and University Medical Center Groningen, Groningen, The Netherlands

${ }^{2}$ University Medical Center Groningen, Center for Development and Innovation

${ }^{3}$ Lifelines cohort study, Groningen, The Netherlands

${ }^{4}$ Department of Obstetrics and Gynecology, University of Groningen and University Medical Center Groningen, Groningen, The Netherlands

${ }^{5}$ Department of Midwifery Science, Amsterdam University Medical Centre, Vrije Universiteit Amsterdam, AVAG/Amsterdam Reproduction and Development, Amsterdam, The Netherlands

${ }^{6}$ Department of Health Sciences, University of Groningen and University Medical Center Groningen, University of Groningen, Groningen, The Netherlands

${ }^{7}$ Department of Paediatric Pulmonology and Paediatric Allergology, Beatrix Children's Hospital, University of Groningen, University Medical Center Groningen, Groningen, The Netherlands.

${ }^{8}$ Department of Pediatrics, University of Groningen and University Medical Center Groningen, Groningen, The Netherlands ${ }^{9}$ Department of Midwifery Science, Amsterdam University Medical Center, Vrije Universiteit Amsterdam AVAG/Amsterdam Public Health and Department of General Practice \& Elderly Care Medicine, University Medical Center Groningen, section Midwifery Science AVAG, University of Groningen, 9700 RB, Groningen, the Netherlands. 


\section{Supplementary Files}

This is a list of supplementary files associated with this preprint. Click to download.

- SupplementarytablesmotherinfanttransmissionbacterialvirusesNM.xlsx 